\title{
THE INFLUENCE OF ARGON ION BOMBARDMENT ON THE ELECTRICAL AND OPTICAL PROPERTIES OF CLEAN SILICON SURFACES
}

\author{
J.W.D. MARTENS, W.F. VAN DEN BOGERT and A. VAN SILFHOUT \\ Department of Applied Physics, Twente University of Technology, P.O. Box 217, \\ 7500 AE Enschede, The Netherlands
}

Received 1 September 1980 ; accepted for publication 24 November 1980

The effect of low energy noble gas ion bombardment on the electrical and optical properties of $\mathrm{Si}(211)$ surfaces has been investigated by surface conductivity and field effect measurements, ellipsometry and AES. With this combination of techniques, information is obtained concerning the electrical properties, the chemical composition and the damage of the surface layer. Upon ion bombardment in the energy range of $500-2000 \mathrm{eV}$, ellipsometry shows the formation of a damaged surface layer with optical properties close to those of an evaporated amorphous silicon film. In order to measure the conductivity changes as sensitive as possible, nearly intrinsic silicon crystals were used. For the clean, $5200 \Omega \mathrm{cm} \mathrm{Si(211)} \mathrm{surface,} \mathrm{bombarded} \mathrm{only} \mathrm{with} \mathrm{a}$ mass-analyzed argon ion beam, a small increase in conductivity is found to occur after a small ion dose (saturation after $5 \times 10^{14}$ ions $\mathrm{cm}^{-2}$ while after $5 \times 10^{13}$ ions $\mathrm{cm}^{-2}$ already half of the increase has occurred). The effect was found to be independent of ion energy between 500 and $2000 \mathrm{eV}$. As the field effect signal did not change after this treatment, it is concluded that the surface state density in the neighbourhood of the Fermi level shows a slight decrease.

\section{Introduction}

Low energy noble gas ion bombardment is a widely used cleaning technique in surface studies. Ion bombardment is known to alter the long range atomic order at the surface [1]. Since on semiconductor surfaces the surface state properties are related to atomic order [2], ion bombardment of semiconductor surfaces may also influence surface state properties.

Only a few investigations on the relation between ion bombardment and surface state properties for the clean silicon surface have been reported so far [3-6], all using relatively ill-defined bombardment conditions and often not well defined crystallographic orientations of the surface.

The influence of energetic ion bombardment on the structure of the silicon lattice has been studied by many authors, using different experimental techniques, such as LEED, RIIEED, X-ray diffraction, TEM, RBS, ESR and IR absorption [7-20]. Recently, ellipsometry has also been used [21-23].

In the present work the interaction of a clean, spatially and energetically well- 
defined noble gas ion beam with clean silicon (211) surfaces has been studied using in-situ ellipsometry, conductivity and field-effect measurements. The changes in the ellipsometric parameters $\Delta$ and $\psi$, which are determined by the optical properties of the reflecting substrate, are interpreted in terms of the build-up of lattice damage upon ion bombardment and are expressed in terms of the complex refractive index of the damaged surface layer $[24,25]$. Field effect and surface conductivity measurements have been carried out to obtain information about the density of surface states near the Fermi-level and the band bending for the clean silicon (211) surface. From changes in field effect mobility and surface conductivity upon noble gas ion bombardment compared to the damage build-up in the surface layer of silicon, a mechanism is deduced to explain the observed effect.

\section{Experimental}

The silicon samples, placed at our disposal by Philips Research Laboratories Eindhoven, were intended to be $\{111\}$ oriented. However, a check experiment in a LEED apparatus and subsequent $\mathrm{X}$-ray diffraction measurements revealed the samples to be $\{211\}$ oriented. The samples, dimensions $30 \times 10 \times 0.3 \mathrm{~mm}$ and resistivity $5200 \Omega \mathrm{cm}$, were polished mirror-like with diamond paste $(\sim 0.1 \mu \mathrm{m})$ and cleaned, prior to deposition of the electrical contacts, in a number of peroxide containing solutions [26]. For the conductivity measurements four titanium contacts were evaporated on the sample surface through a tantalum mask.

Irrespective of heat treatment, these contacts yielded always a linear currentvoltage behaviour. With AES, no surface diffusion could be observed after $5 \mathrm{~min}$ at $1200^{\circ} \mathrm{C}$. Within the experimental error no differences in resistance were observed between measurements with two or with four contacts.

The experiments were carried out in two UHV systems. System I consists of a RIBER-UNI-7 pumping unit with integrated $400 \mathrm{l} / \mathrm{s}$ ion getter pump and sublimator with cryoshield, and a specially designed all-metal vacuum chamber provided with facilities for surface conductivity, field effect measurements and ion bombardment. A turbomolecular pump is available for pumping. First to prevent a glow discharge when starting the ion getter pump, which influences the surface state density of the silicon samples noticeably and secondly for pumping during gas exposure and ion bombardment allowing a base pressure $<5 \times 10^{-7} \mathrm{~Pa}$. Over $90 \%$ of this pressure consisted of noble gases and methane, backstreaming from the ion pump. Oxygen (purity $99.995 \%$ ) and argon (99.999\%) can be separately introduced into the vacuum chamber or into the ionization chamber of the ion gun.

After bake-out at $200^{\circ} \mathrm{C}$ the base pressure was below $4 \times 10^{-9} \mathrm{~Pa}$, being the $\mathrm{X}$-ray limit of the nude Bayard-Alpert gauge (Granville Philips 274-022). A Riber QMM16 quadrupole was available for residual gas analysis. The ion gun was a modified NODUS ion gun [27] and consisted basically of an ionization chamber, a Wien type velocity filter and a final focussing lens. The gun was capable of producing 


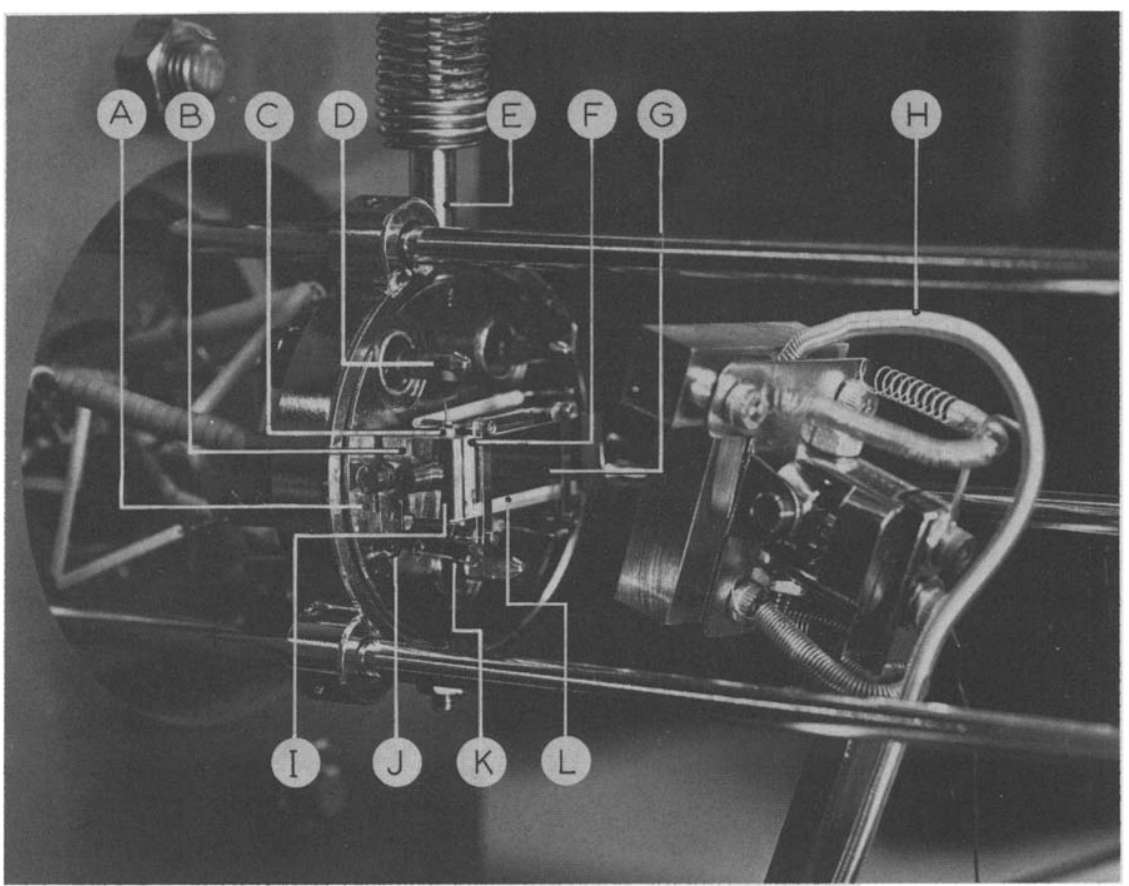

Fig. 1. Photograph of the sample holder: (A) base plate; (B) stainless steel sample support; (C) heater filament; (D) adjustment screw for the fieldeffect electrode distance; (E) rotational axis and coupling to rotary feed through; $(F)$ outer titanium contact; $(G)$ sample; $(H)$ co-axial voltage cable; (I) molybdenum sample clamp; (J) thermocouple; (K) one of the inner contact connections; (L) $\mathrm{Al}_{2} \mathrm{O}_{3}$ sample support.

an ion beam of up to $3 \times 10^{-7} \mathrm{~A}$ in the energy range $100-2500 \mathrm{eV}$ with an operating pressure of $\sim 1 \times 10^{-4} \mathrm{~Pa}$ in the ionization region. A small diaphragm (diameter $2 \mathrm{~mm}$ ) separates this region from the main vacuum chamber. Bombardments were performed at normal incidence.

The sample was mounted on a home-made sample holder which allows a translation of $2-3 \mathrm{~mm}$ in three directions and a rotation of $\pm 45^{\circ}$ (around the vertical axis). The sample can be heated to $\sim 300^{\circ} \mathrm{C}$ by means of two tungsten filaments located in the two sample supports. Higher temperatures, up to $1200^{\circ} \mathrm{C}$, were achieved by passing a dc current through the sample. Below $250^{\circ} \mathrm{C}$ the temperature was measured by a chromel/alumel thermocouple clamped to one of the sample supports. Temperatures above $250^{\circ} \mathrm{C}$ were measured with an optical pyrometer (IRCON T5C). The sample temperature could be regulated to $\pm 0.1^{\circ} \mathrm{C}$ in the range $20-100^{\circ} \mathrm{C}$ by means of a suitable controller.

For field effect measurements a flat T-shaped stainless-steel electrode assembly could be rotated around a horizontal axis and placed in front of the sample (see fig. 1). The parallelism of this assembly with respect to the sample surface was 


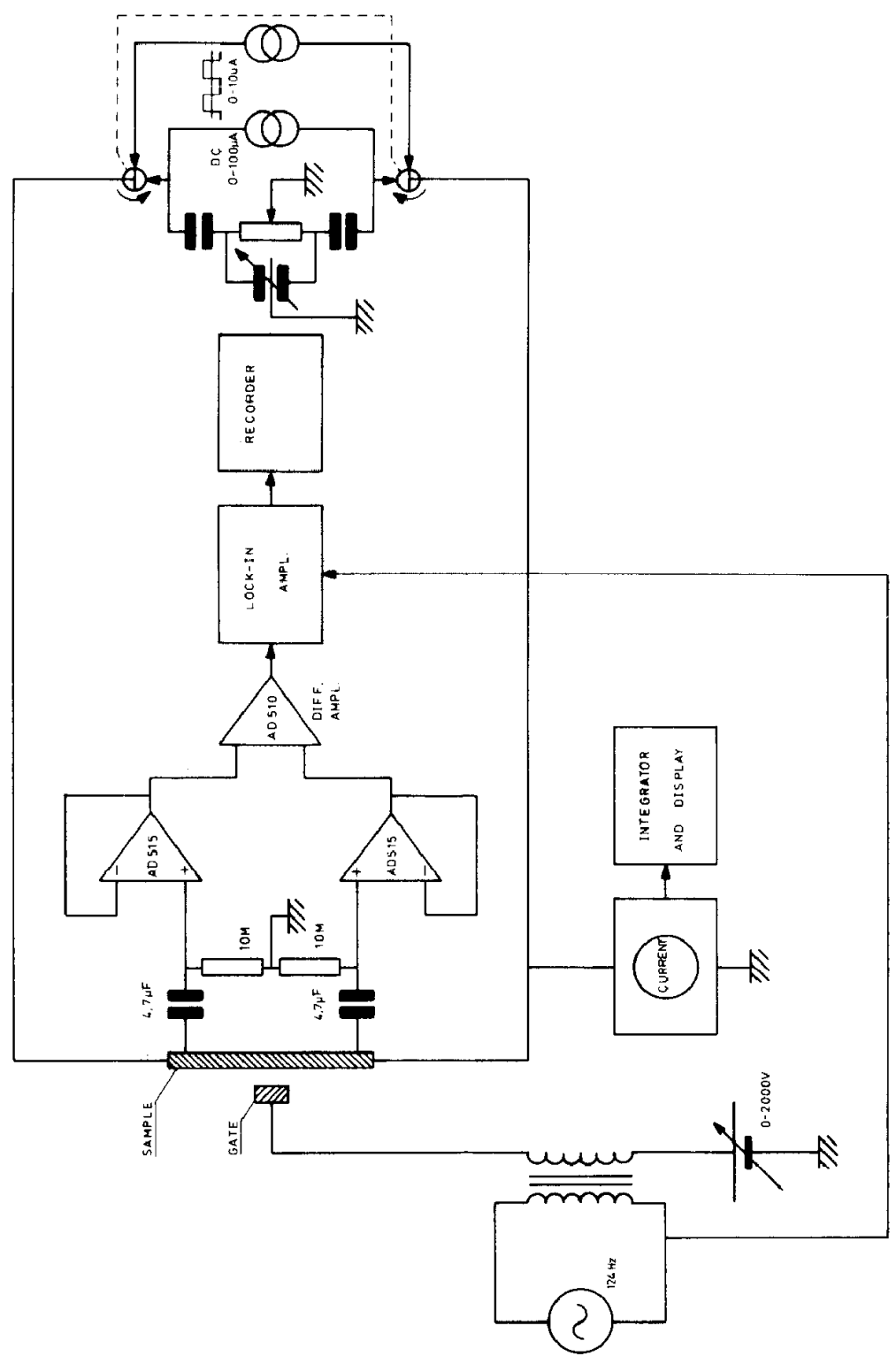


adjusted by three screws located at positions around the sample and can be checked optically in air. Within the T-shaped electrode a second, flat square $(1 \times 1 \mathrm{~cm})$ electrode was positioned. The actual modulation voltage was applied only to the inner square electrode and the $\mathrm{T}$-shaped electrode was grounded to shield the sample from any unwanted electrical fields. The distance between electrode and sample is about $100 \mu \mathrm{m}$. Its value was determined from the capacitance value $\left(\sim 10^{-11}\right.$ $\mathrm{F} / \mathrm{cm}^{2}$ ). The sample resistance and field effect were measured according to fig. 2 , by using the four titanium contacts deposited on the sample. For resistance measurements an ac constant current source was connected to the sample at the outer contacts, while the resulting potential at the inner contacts was detected by means of a lock-in amplifier. In this way relative resistance changes of $1: 10^{5}$ can be delected. The absolute accuracy was $\pm 0.05 \%$. For field effect measurements a dc constant current source is used, while an ac voltage is applied to the field effect electrode.

The UHV system II is of similar design as system I, and is equipped with facilities for ellipsometry, AES, ion bombardment and resistance measurements. A schematic of this system is given in fig. 3. The sample holder was mounted on a modified VG-UMD-1 manipulator. The sample can be cooled down to liquid nitrogen temperature and can be heated to $\sim 300^{\circ} \mathrm{C}$ by passing a current through the $\mathrm{LN}_{2}$ entrance capillary of the sample holder while flushing it with an inert gas. By using a suitable controller, the sample temperature was stabilized within $\pm 0.05^{\circ} \mathrm{C}$. A VG-HCMA was used for AES. The samples were initially cleaned by

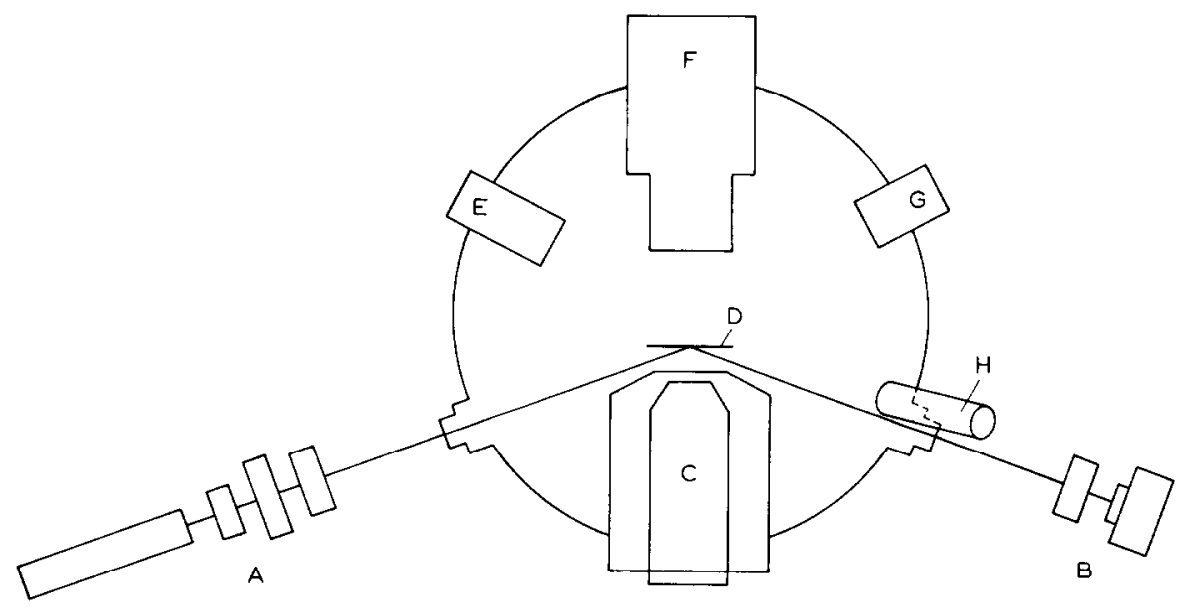

Fig. 3. Top view cross section of UHV system II: (A, B) ellipsometer set-up; (C) Auger analyser with electron gun; (D) sample; (E) oxygen leak valve; (F) quadrupole mass spectrometer; (G) ionization gauge; $(\mathrm{H})$ glancing incidence ion gun. 
means of a glancing incidence cold-cathode ion gun (VG-AG 2). The angle of incidence of the ion beam is $75^{\circ}$ with respect to the surface normal. The ion gun is operated at a pressure of $\sim 8 \times 10^{-4} \mathrm{~Pa}$ and produces a target current of up to $2 \times 10^{-6} \mathrm{~A} \mathrm{~cm}^{-2}$ at an energy of $2000 \mathrm{eV}$. For comparison with the electrical measurements in system I in some later experiments, the HCMA has been replaced by the NODUS ion gun from system I and the bombardment was carried out with this mass selective gun at normal incidence. A more detailed description of the experimental systems is given in ref. [28].

In both systems the ion dose is obtained from the integrated sample current. No suppression for secondary electrons is used because the other experimental techniques required easy sample excess. However, a correction for this effect was applied according to Hagstrum $[28,29]$.

The home-made automatic ellipsometer was of the self-nulling type with the compensator preceding the sample. The polarizer as well as the analyser were connected to stepping motors and could be read to $\pm 0.005^{\circ}$. A Hughes 3225 H.PC $\mathrm{He}-\mathrm{Ne}$ laser $(\lambda=632.8 \mathrm{~nm})$ was used as light source and a silicon photocell (Siemens BPY 63) was applied as detector. A reading for $\mathbf{P}$ and $\mathbf{A}$ was obtained by averaging the readings for equal intensity on both sides of the minimum and takes about $15 \mathrm{~s}$. In this way transient phenomena as encountered upon ion bombardment can be easily followed. All measurements were performed in zone 1 [30]. Usually a series of four zone measurements was performed before and after the ion bombardment experiment to calibrate changes in $\Delta$ and $\psi$ obtained in zone 1 . This yields the calibration of the relative changes in $\Delta$ and $\psi, \delta \Delta$ and $\delta \psi$. Absolute values of $\Delta$ and $\psi$ were obtained after a correction for the optical window birefringence. This correction was determined afterwards by a series of four zone measurements with clean and contaminated surfaces in vacuum and at $1 \mathrm{~atm}$ and with contaminated surfaces with and without optical windows.

\section{Results}

\subsection{Sample treatment}

Although the contact properties remain unaltered upon heat treatment, the room temperature conductance of the silicon samples increased strongly after heating in UHV. The main cause appeared to be diffusion of a boron surface contamination into the crystal, resulting in a highly conductive p-type layer. The identification of boron in the sub-surface layer was achieved by means of SIMS. After heating at $900^{\circ} \mathrm{C}$ for one hour, this showed a steep concentration gradient over three decades in a layer of $30 \mathrm{~nm}$ starting with $\sim 10^{19}$ atoms $/ \mathrm{cm}^{3}$ at the surface.

After replacing the borosilicate glass viewports of the UHV system by fused quartz components, heating at temperatures above $\sim 800^{\circ} \mathrm{C}$ caused a similar resistance behaviour. This second effect consists of an increase of $n$-type conduc- 
tivity caused by radiation quenching of the sample temperature $[5,31-33]$. On the $5200 \Omega \mathrm{cm}$ samples used here, this effect could be avoided by limiting the annealing temperature to $800^{\circ} \mathrm{C}$.

In order to eliminate the influence of the back surface the samples were first annealed at $800^{\circ} \mathrm{C}$ until their room temperature conductance reached a constant value. The front surface was finally cleaned by a series of argon ion bombardment and annealing cycles at $800^{\circ} \mathrm{C}$. This temperature has been shown to be sufficient to restore surface damage created by low-energy ion bombardment $[1,7,8]$. Also previously implanted argon ions are completely driven out of the sample at temperatures already below $800^{\circ} \mathrm{C}[7,28]$.

\subsection{Electrical effects of mass-analyzed argon ion bombardment on Si(211) surface}

Field effect measurements indicate the presence of a n-type surface layer on the etched, uncleaned $5200 \Omega \mathrm{cm}$ silicon samples, in agreement with literature data $[3,34]$. The absolute value of the field effect mobility $\mu_{\mathrm{FE}}$, defined as

$\mu_{\mathrm{FE}}=-\mathrm{d} \Delta \sigma_{\mathrm{s}} / \mathrm{d} Q_{\text {ind }}$,

where $\Delta \sigma_{\mathrm{s}}$ is the surface conductivity and $Q_{\text {ind }}$ the total induced charge per unit area, ranged between 0.2 and $1 \mathrm{~cm}^{2} \mathrm{~V}^{-1} \mathrm{~s}^{-1}$ for different crystals.

After cleaning the samples by a series of $\mathrm{Ar}^{+}$bombardment and annealing cycles finally a constant overall conductance was reached at a lower value as compared to the etched surface. The value of $\mu_{\mathrm{FE}}$ had decreased to $\sim 0.1 \mathrm{~cm}^{2} \mathrm{~V}^{-1} \mathrm{~s}^{-1}$ (still n-type). AES measurements in system II revealed an atomically clean surface after

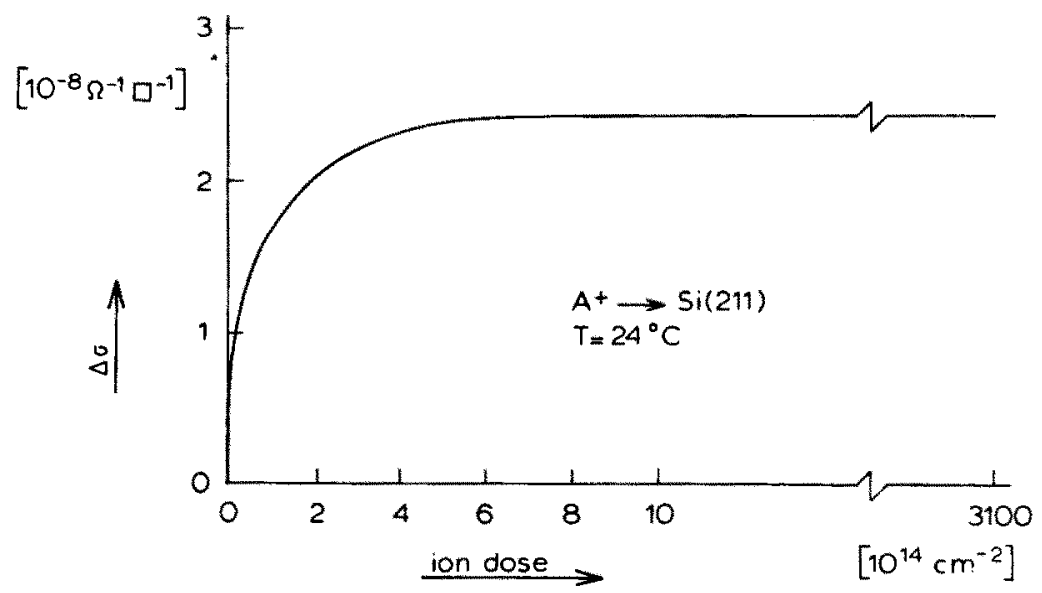

Fig. 4. Conductivity increase upon bombarding the clean $\operatorname{Si}(211)$ surface with argon ions in the energy range $500-2000 \mathrm{eV}$ yersus ion dose. 


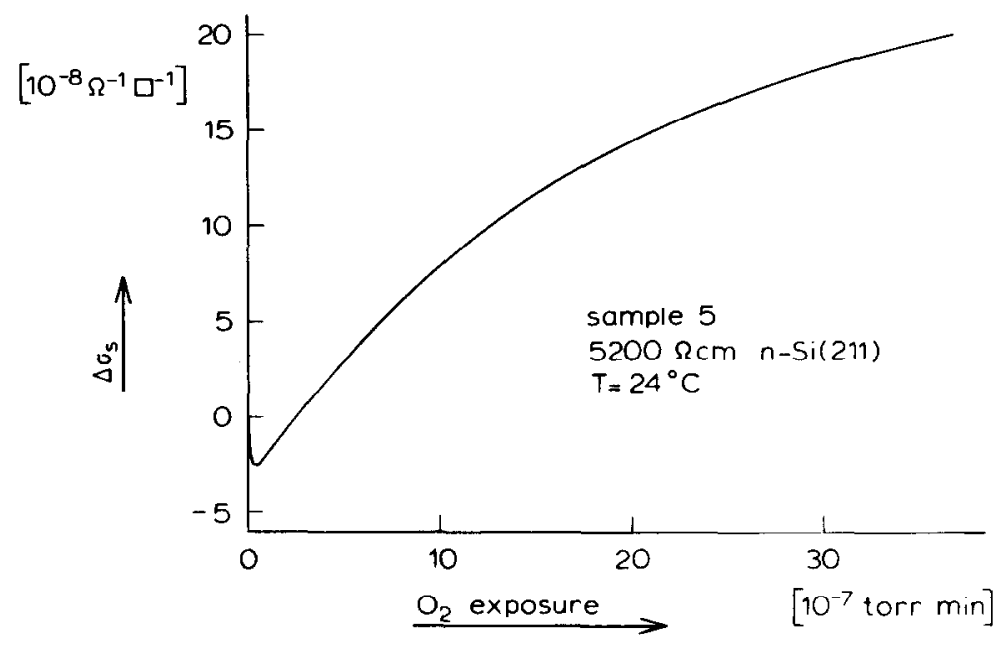

Fig. 5. Conductivity changes upon oxygen adsorption on the clean Si(211) surface versus oxygen exposure.

a similar treatment. The resulting conductivity changes upon $\mathrm{Ar}^{+}$bombardment at normal incidence on such a silicon (211) surface are given in fig. 4, where the conductivity increase is plotted versus the incident ion dose. The current density varied between $0.2 \times 10^{-7}$ and $1 \times 10^{-7} \mathrm{~A} \mathrm{~cm}^{-2}$ in successive experiments. No influence of current density has been observed. Argon ion bombardment leads to a small conductivity increase which saturates already after a very small $\left(\sim 0.5-1 \times 10^{15}\right.$ ions $\mathrm{cm}^{-2}$ ) ion dose. The same results are obtained irrespective of ion energy between 500 and $2000 \mathrm{eV}$. The value of $\mu_{\mathrm{FE}}$ after $\mathrm{Ar}^{+}$bombardment remains unaltered $\left(0.1 \mathrm{~cm}^{2} \mathrm{~V}^{-1} \mathrm{~s}^{-1}\right)$ as compared to the unbombarded surface.

Oxygen adsorption on a clean, annealed surface caused a change in conductivity, given in fig. 5. As will be discussed below this change confirms the presence of an n-type surface layer on the clean surface. To check the influence of the back surface the adsorption of oxygen on a surface that had only been annealed was studied. In contrast with fig. 5 the conductivity showed no minimum and a smaller increase.

\subsection{Optical effects of $\mathrm{Ar}^{+}$bombardment on Si(211) surface}

From the measured changes $\delta \Delta$ and $\delta \psi$, defined as $\delta \Delta=\Delta-\bar{\Delta}$ and $\delta \psi=\psi-\bar{\psi}$, where $\bar{\Delta}$ and $\bar{\psi}$ refer to the clean substrate and $\Delta$ and $\psi$ to the substrate covered with a layer, the build-up of damage in the surface region of the sample upon $\mathrm{Ar}^{+}$ bombardment was determined in UHV system II. The bombardments were performed at normal incidence with $2000 \mathrm{eV} \mathrm{Ar}{ }^{+}$ions. After several cycles of bom- 


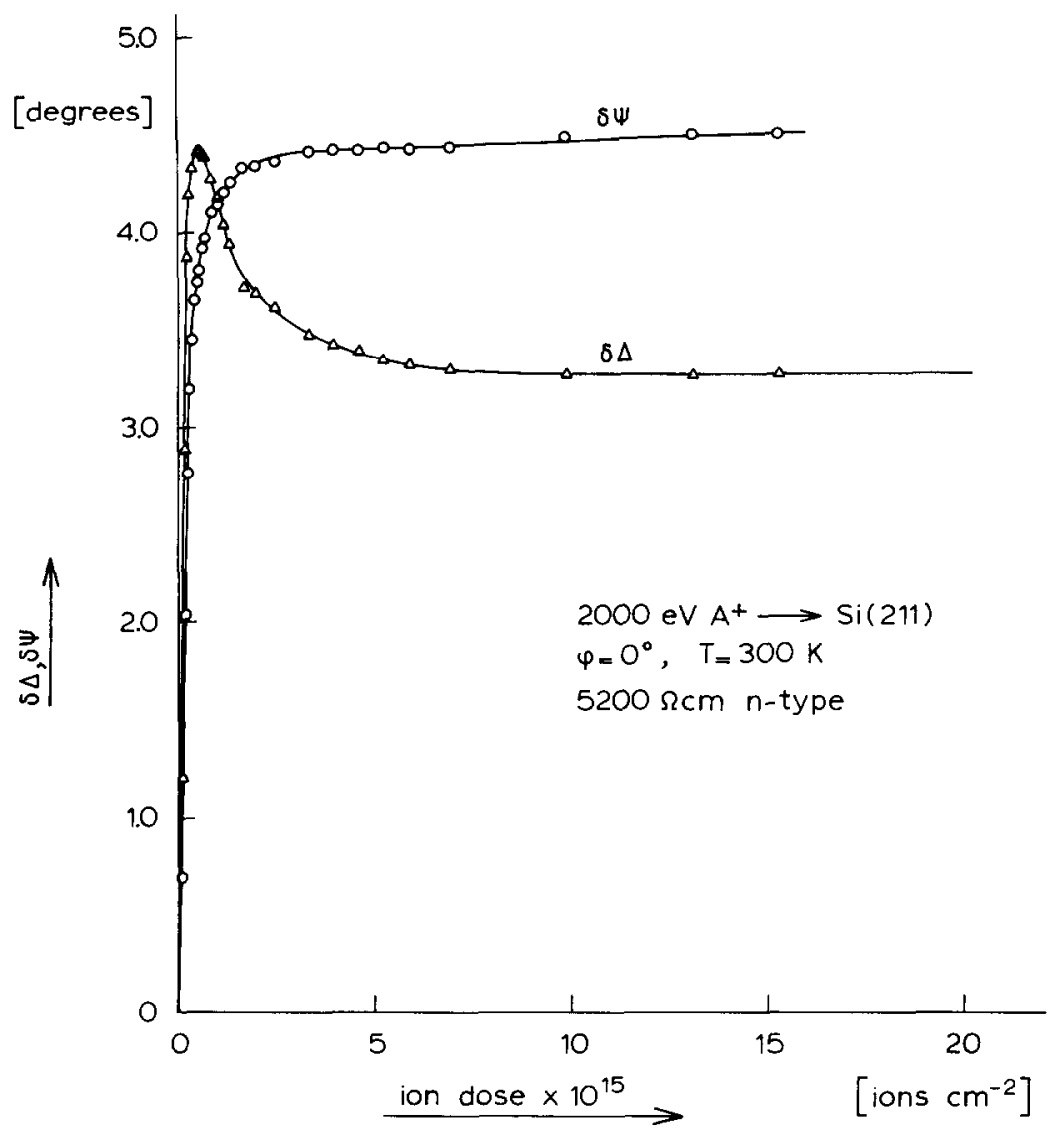

Fig. 6. Changes in the ellipsometric parameters $\Delta$ and $\psi$ upon $2000 \mathrm{eV}$ argon ion bombardment at normal incidence at $300 \mathrm{~K}$.

bardment and annealing, at a cumulative ion dose of $\sim 1 \times 10^{17}$ to $1 \times 10^{18}$ ions $\mathrm{cm}^{-2}$ depending on the pretreatment, the values of $\Delta$ and $\psi$ for the clean and undamaged silicon surface became constant, corresponding with $\tilde{n}=3.88-\mathrm{i} 0.059$. This value is in reasonable agreement with literature data: $\tilde{n}=3.90-i 0.02$ [22] or $\tilde{n}=3.875-\mathrm{i} 0.023[35]$.

The results of the ellipsometric measurements for $2000 \mathrm{eV} \mathrm{Ar}^{+}$bombardment at normal incidence are shown in fig. 6 . The current density was $1.4 \times 10^{-7} \mathrm{~A} \mathrm{~cm}^{-2}$ corrected for secundary electron emission. It should be noted that $\delta \Delta, \delta \psi$ gradually approach a saturation value at an ion dose which is considerably higher than for the electrical effects (fig. 4). 


\section{Discussion}

From the measured changes $\delta \Delta$ and $\delta \psi$ values of the complex refractive index of the damaged surface layer have been computed by assuming a simple model with a homogeneously damaged layer with a thickness of $5 \mathrm{~nm}$. The layer thickness has been estimated from the results of the computer program "Marlowe" based on a model of ion ranges in solids introduced by Robinson and Torrens [36]. In this model the incident particle is followed during the collision sequence in an ordered array of atoms and the results of primary particles incident at random positions are averaged to obtain a range distribution. In the approach of Lindhard, Scharff and Schiptt [37] (LSS theory) the solid is regarded as a highly compressed gas and a different potential model (Thomas-Fermi potential instead of a Molière potential as used by Robinson and Torrens) has been used. In fig. 7 the results of the "Marlowe" calculations are shown and also the LSS calculated argon distribution is marked. The ellipsometric parameters include the following effects caused by ion bombardment:

(a) - surface damage: roughness, defects,

- implantation of argon ions (probably negligible);

(b) - bulk damage (main effect).

The optical information depth $(\sim 1.5 \mu \mathrm{m}$ at $\lambda=632.8 \mathrm{~nm}$ and $300 \mathrm{~K})$ is much larger than the ion range in the substrate.

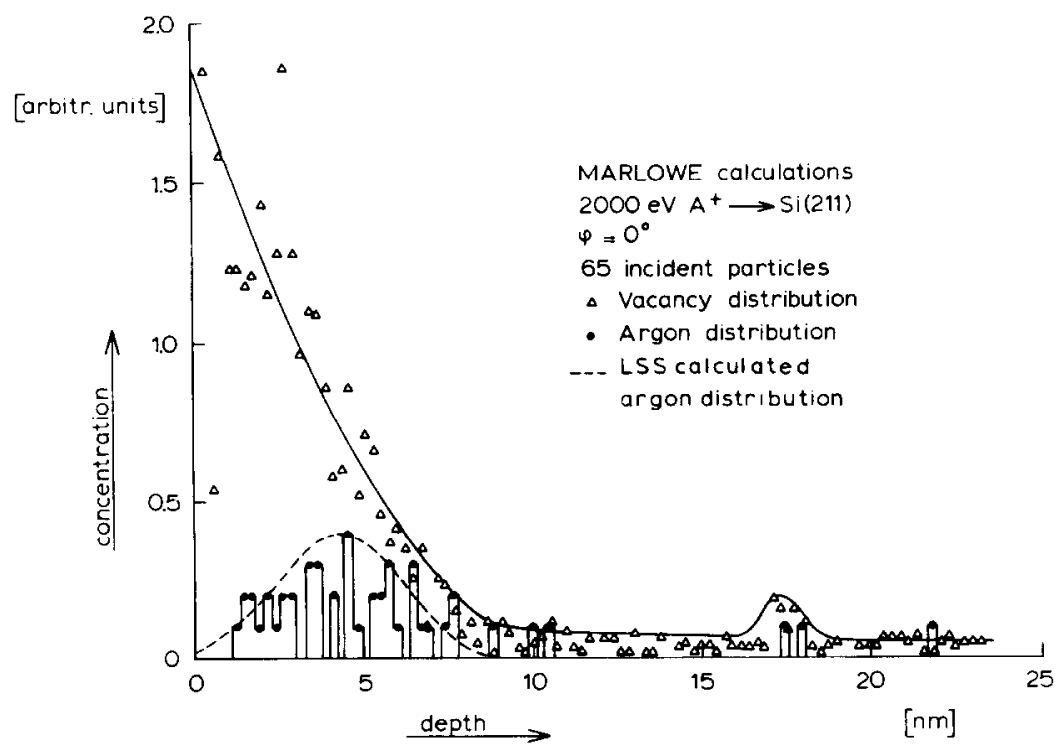

Fig. 7. Range distribution of $2000 \mathrm{eV}$ argon ions incident at normal incidence on a Si(211) plane. Also indicated are the vacancy distribution and the LSS calculated primary particle distribution. 
For the stationary state the values of $\delta \Delta$ and $\delta \psi$ correspond to a complex refractive index for the damaged surface layer of $\tilde{n}=4.84-\mathrm{i} 1.54$. Calculations show that the maximum in the $\delta \Delta$ curve (fig. 6 ) does not correspond to a discontinuity in the layer properties, as has also been reported by Nakamura et al. [22] for higher ion energies. In our simplified model the refractive index of the layer for the maximum in $\delta \Delta$ is calculated to be $\tilde{n}=4.78-\mathrm{i} 1.31$.

These qualitative results, i.e. an increase in both the real and imaginary part of the complex refractive index, correspond to those of Ibrahim and Bashara [21]. The effective refractive index of the damaged layer may be compared to that of amorphous, evaporated silicon films [38], which show similar deviation from the refractive index of crystalline silicon.

The strong difference in saturation ion dose in the optical and the conductivity measurements (figs. 6 and 4), determined under comparable conditions and the insensitivity of the electrical effects to ion energy, indicate that no direct relation exists between the overall lattice damage as measured optically and the changes in electrical conductivity. The changes in conductivity found upon $\mathrm{Ar}^{+}$bombardment are therefore attributed to changes in the surface space charge layer, i.e. in the surface conductivity. By definition the surface conductivity $\Delta \sigma_{\mathrm{s}}$ is given by

$\Delta \sigma_{\mathrm{s}}=e\left(\mu_{\mathrm{ns}} \Delta N+\mu_{\mathrm{ps}} \Delta P\right)$,

where $e$ is the elementary charge, $\mu_{\mathrm{ns}}$ and $\mu_{\mathrm{ps}}$ are the effective carrier mobilities at the surface and $\Delta N, \Delta P$ the excess electron and hole concentrations per unit surface area [39]. The values of $\Delta \sigma_{\mathrm{s}}$ can be calculated as a function of the band bending at the surface $k T v_{\mathrm{s}}$, where $v_{\mathrm{s}}$ is defined as in fig. 8 . The results of these calculations

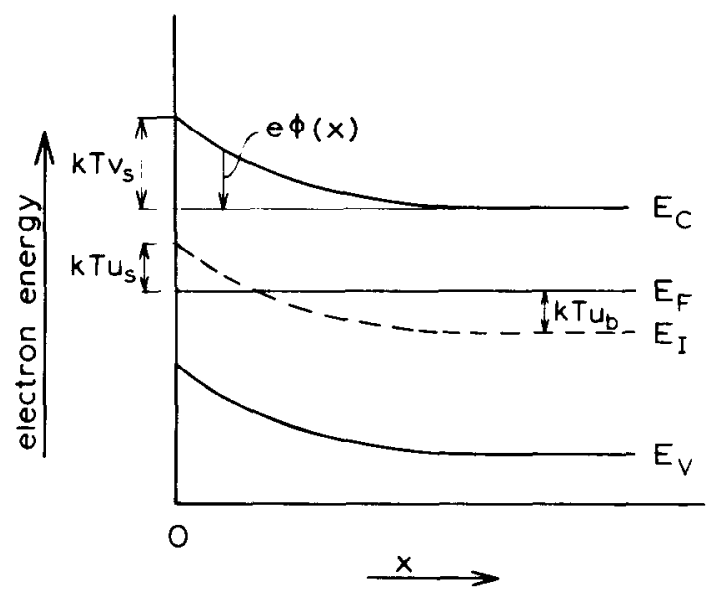

Fig. 8. An energy diagram in the surface space charge layer and in the bulk, for a n-type semiconductor. 


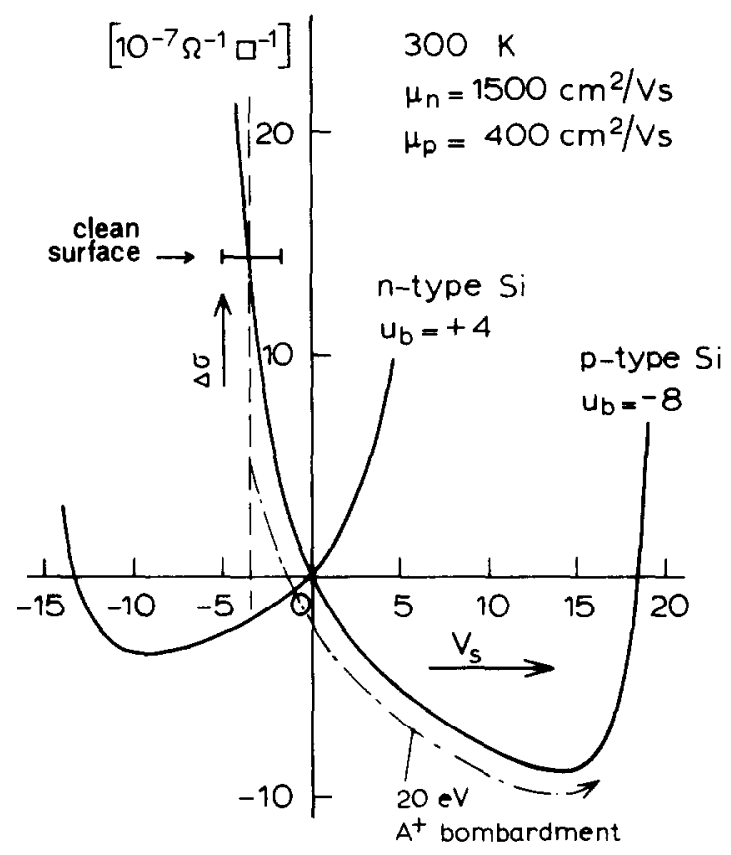

Fig. 9. Calculated curves of the surface conductivity versus band bending at the surface $v_{\mathbf{s}}$. Indicated are the position of clean surface and the direction of changes in $v_{3}$ upon ion bombardment for p-type silicon [3].

are given in fig. 9 for $5200 \Omega \mathrm{cm}$-type and $1000 \Omega \mathrm{cm}$ p-type silicon at $300 \mathrm{~K}$; in view of the small band-bending bulk mobilities have been used. Also indicated is the value of $v_{s}$ for the clean surface obtained on $1000 \Omega \mathrm{cm}$-type silicon by Heiland and Lamatsch [3]. The latter result was obtained for a cylindrical crystal with the axis parallel to the [111] direction. Our results for n-type $\mathrm{Si}$ indicate always a value of $v_{s}$ corresponding to the right hand branch of the calculated curve. Starting from the etched surface at a relatively high value of $\Delta \sigma_{s}\left(\sim 0.5-2 \times 10^{-6} \Omega^{-1} \square^{-1}\right.$ for successive samples), $\mathrm{Ar}^{+}$bombardment and annealing decreases the value of $v_{\mathrm{s}}$ and $\Delta \sigma_{\mathrm{s}}$. However, the clean surface remains n-type.

Oxygen adsorption, showing an increase of $v_{s}[3,4]$ leads to an increase in conductivity of our samples whereas on p-type bulk silicon a decrease has been found $[3,4]$. Comparing our results with those of Boonstra [4] for p-type silicon, we may conclude to the presence of a $n$-type surface layer on the clean surface of our n-type Si crystals.

The argon ion induced conductivity changes correspond then to a very small change in $v_{\mathrm{s}}$ of the order of $1-2 k T$ (in positive direction). At the same time the field effect data indicate a slight decrease in surface state density near the Fermi 
level. No information can be obtained on changes in surface state density at relatively large distance from the Fermi level, as observed e.g. by Rowe and Ibach by using ELS [40]. Interpreted in changes of $v_{s}$, the conductivity changes found upon unanalyzed $\mathrm{Ar}^{+}$bombardment by Heiland and Lamatsch [3] require a much larger change in $v_{\mathrm{s}}$ (indicated in fig. 9). Measurements with a mass-unanalyzed ion beam on our samples have shown an increase of up to $1 \times 10^{-5} \Omega^{-1} \square^{-1}$ in conductivity [28]. Since our results indicate the necessity of the use of a mass-analyzed ion beam, the difference between our measurements and those of Lamatsch may be attributed to the difference in surface orientation and to impurities. $\Lambda$ detailed description of the electrical and optical effects upon unanalyzed ion bombardment will be given elsewhere.

Careful $\mathrm{Ar}^{+}$bombardment produced on our clean $5200 \Omega \mathrm{cm} \mathrm{n}$-type silicon surfaces a small change in $\Delta \sigma_{\mathrm{s}}$, corresponding to a small change in band bending at the surface $v_{\mathrm{s}}$. Eq. (2) indicates that $\Delta \sigma_{\mathrm{s}}$ is composed of the product of a mobility and an excess carrier concentration. In general lattice damage will reduce the carrier mobility. However, our experimental results show an increase in conductivity, that already saturates while the damage still increases, as evident from ellipsometry (figs. 4, 6). Apparently mobility effects are not of prime importance in our case. This is understandable in view of the small band bending at the surface, causing a relatively extended space charge region $(\sim 1 \mu \mathrm{m})$ at one hand and the small damaged layer thickness as predicted by "Marlowe" at the other [39].

\section{Acknowledgements}

The authors wish to thank Professor G.A. Bootsma, Professor H.H. Brongersma and Professor M.J. Sparnaay for many stimulating discussions, Dr. G.E. Thomas of Philips Research Laboratories for performing the "Marlowe" calculations and Dr. A.E. Morgan of the same laboratory for the SIMS measurements.

\section{References}

[1] R.E. Schlier and H.E. Farnsworth, J. Chem. Phys. 30 (1959) 917;

J.J. Lander, G.W. Gobeli and J. Morrison, J. Appl. Phys. 34 (1963) 2298;

F. Jona, IBM J. Res. 9 (1965) 375;

R.J, Fortner, Bull. Am. Phys. Soc. 18 (1973) 56.

[2] F. Bäuerle, W. Mönch and M. Henzler, J. Appl. Phys. 43 (1972) 3917.

[3] G. Heiland and H. Lamatsch, Surface Sci. 2 (1964) 18;

H. Lamatsch, Phys. Status Solidi (a) 9 (1965) 19.

[4] A.H. Boonstra, Philips Res. Rept. 3 (1968).

[5] J.T. Law, J. Phys. Chem. Solids 14 (1960) 9.

[6] B.A. Nesterenko and O.V. Snitko, Surface Sci. 5 (1966) 380;

B.A. Nesterenko, O.V: Snitko and V.T. Rozumnyuk, Surface Sci. 9 (1968) 407.

[7] J.C. Bean, G.E. Becker, P.M. Petroff and T.E. Seidel, J. Appl. Phys. 48 (1977) 907. 
[8] T. Ichikawa and S. Ino, Japan. J. Appl. Phys. 17 (1978) 1675.

[9] A.I. Gerasimenko, E.I. Zorin, P.V. Pavlov and D.I. Tetel'baum, Phys. Status Solidi (a) 12 (1972) 679.

[10] P.V. Pavlov and D.I. Tetel'baum, Soviet Phys.-Dokl. 12 (1968) 746.

[11] A.I. Gerasimenko, E.I. Zorin, P.V. Pavlov and D.I. Tetel'baum, Soviet Phys.-Dokl. 15 (1970) 430.

[12] P.V. Pavlov, Soviet Phys. Cryst. 18 (1974) 506.

[13] N.A.G. Ahmed, C.E. Christodoulides and G. Carter, Radiation Effects 38 (1974) 506;

N.A.G. Ahmed, C.E. Christodoulides and G. Carter, Phys. Letters 69A (1979) 431.

D.A. Thompson, R.S. Walker and J.A. Davies, Radiation Effects 32 (1977) 135.

[14] S.T. Picraux and F.L. Vook, Radiation Effects 11 (1971) 179.

[15] N.A. Sobolev, G. Götz, W. Karthe and B. Schnabel, Radiation Effects 42 (1979) 23.

[16] G. Götz, W. Karthe, B. Schnabel and N. Sobolev, Phys. Status Solidi (a) 50 (1978) 209.

[17] M.H. Brodsky and R.S. Title, Phys. Rev. Letters 23 (1969) 581.

[18] H.J. Stein, F.L. Vook and J.A. Borders, Appl. Phys. Letters 14 (1969) 328;

J.W. Corbett, Radiation Effects 6 (1970) 3;

F.L. Vook and H.J. Stein, Radiation Effects 6 (1970) 11.

[19] E.K. Baranova, V.M. Gusov and I.B. Khaibullin, Soviet Phys.-Semicond. 9 (1975) 630 .

[20] E.K. Baranova, Soviet Phys.-Semicond. 9 (1975) 763.

[21] M.M. Ibrahim and N.M. Bashara, Surface Sci. 30 (1972) 632.

[22] K. Nakamura, R. Gotoh and M. Kamoshida, J. Appl. Phys. 50 (1979) 3985;

K. Nakamura and M. Kamoshida, Radiation Effects 42 (1979) 29.

[23] K. Watanabe, M. Miyao, I. Takemoto and N. Hashimoto, Appl. Phys. Letters 34 (1979) 518 ;

T. Motooka and K. Watanabe, J. Appl. Phys. 51 (1980) 4125.

[24] H. Albers, J.M.M. Droog and G.A. Bootsma, Surface Sci. 64 (1977) 1.

[25] D.E. Aspnes and A.A. Studna, Surface Sci. 96 (1980) 294.

[26] R.L. Meek, J. Electrochem. Soc. 120 (1973) 1241.

[27] H.H. Brongersma, N. Hazewindus, J.M. van Nieuwland, A.M.M. Ottens and A.J. Smets, Rev. Sci. Instr. 49 (1978) 707.

The present ion source is a new design of the described ISS system.

[28] J.W.D. Martens, The influence of noble gas ion bombardment on the electrical and optical properties of clean silicon surfaces, Thesis, Twente (1980).

[29] H.D. Hagstrum, Phys. Rev. 114 (1960) 940.

[30] F.L. McCrackin, E. Passaglia, R.R. Stromberg and H.L. Steinberg, J. Res. Natl. Bur. Std. A67 (1963) 363.

[31] M.L. Swanson, Phys. Status Solidi 33 (1969) 721.

[32] G. Bemski and E.A. Dias, J. Appl. Phys. 35 (1964) 2983.

[33] L. Elstner and W. Kamprath, Phys. Status Solidi 22 (1967) 541.

[34] T.M. Buck and F.S. McKim, J. Electrochem. Soc. 105 (1958) 709.

[35] M.A. Hopper, R.A. Clarke and L. Young, J. Electrochem. Soc. 122 (1975) 1216.

[36] M.T. Robinson and J.M. Torrens, Phys. Rev. B9 (1974) 5008.

[37] J. Lindhard, M. Scharff and H.E. Schiфtt, Kgl. Danske Videnskab. Selskab Mat. Fys. Medd. 33 (1963) 3.

[38] R. Gregorovici, Mater. Res. Bull. B3 (1968) 13;

D. Beaglehole and M. Zavetova, J. Non-Crystalline Solids 4 (1970) 272;

D.J. Pierce and W.E. Spicer, Phys. Rev. B5 (1972) 3017.

[39] A. Many, Y. Goldstein and N.B. Grover, Semiconductor Surfaces (North-Holland, Amsterdam, 1965).

[40] J.E. Rowe and H. Ibach, Phys. Rev. Letters 31 (1973) 102. 